\title{
Effects of ThermoMechanical Coupling in Tribological Surface Transformations: A One-Dimensional Modelling Including Irreversible Solid-Solid Phase Transformations and Classical Plasticity
}

\author{
Grégory Antoni \\ Haute Ecole d'Ingénierie et de Gestion du Canton de Vaud, Institut COMATEC, Route de Cheseaux 1, \\ 1401 Yverdon-les-Bains, Switzerland
}

Correspondence should be addressed to Grégory Antoni; antoni.gregory@yahoo.fr

Received 20 November 2012; Revised 1 February 2013; Accepted 1 February 2013

Academic Editor: Jacques Huot

Copyright (C) 2013 Grégory Antoni. This is an open access article distributed under the Creative Commons Attribution License, which permits unrestricted use, distribution, and reproduction in any medium, provided the original work is properly cited.

Under compressive loads combined with friction, some materials undergo Tribological Surface Transformations (TSTs) on the surface of the loaded parts and in the immediately vicinity, which in the case of metals, are known as irreversible solid-solid phase transformations. During the solid-solid phase transformations occurring under mechanical loads, TRansformation Induced Plasticity (TRIP) processes are generated at much lower stress levels than those associated with the yield strength of the material in classical plasticity. In order to assess the effects of thermomechanical coupling in these TSTs, a one-dimensional modelling based on irreversible solid-solid phase transformations and classical plasticity is presented and discussed.

\section{Introduction}

The TSTs which have been affecting some rails of the French railroad for the last twenty years consist of irreversible solidsolid phase transformations resulting in the development of a "White Etching Layer" (WELs) on the rail tread and which grows in depths from several nanometers to more than $100 \mu \mathrm{m}$ [1]. After the passing of some trains, the first "white" phase layers appear on the surface of the rails (which initially have a ferritic-pearlitic structure) before penetrating more deeply, and resulting in a "quasi-surfacic" martensitic transformation, that is, the material composing the rails is transformed into martensite [2]. The initiation and development of TSTs are induced by the combined thermomechanical loads in the wheel/rail contact area, where the normal and tangential stresses are combined with a temperature increase due to the friction occuring in the contact, that is, TSTs result from the thermomechanical coupling processes. During the steel phase transformations which occur under mechanical loads, an anomalous plastic flow known as TRansformation Induced Plasticity (TRIP) is generally superimposed on the classical plastic processes at stress levels which are much lower than the initial strength of the softest phase. Greenwood and Johnson [3] has explained TRIP process by suggesting that the microplasticity generated by the incompatibility between the volumes involved in the phase transformation may be oriented by the external stress applied. Many models have been presented to account for TRIP phenomena [4-8]. In order to assess the thermomechanical coupling associated with TSTs, a one-dimensional version of the model presented [9], which was developed in a thermodynamically consistent framework, is presented in Section 2. In this model, the irreversible phase transformations are taken to result from "strong" thermomechanical coupling, that is, from the combined effects of the change in temperature and the mechanical dissipation. A numerical example is illustrated in Section 3 in the case of a bar subjected to a thermomechanical loading, which is liable to undergo an irreversible solid-solid phase transformation, possibly with the classical plasticity. 


\section{A $1 D$ Phenomenological Modelling Approach Applied to Tribological Surface Transformations (TSTs): Thermomechanical Coupling}

Based on the previous TRIP studies, which are extended here to the thermomechanical coupling associated with TSTs [9], the 1D phenomenological model presented in this Section is developed as follows:

(i) the absolute temperature $T$ is explicitly considered and taken to be a state variable;

(ii) the mass fraction of the daughter phase (i.e., the "white" martensite phase) is:

$$
z=\frac{\rho_{d}}{\rho} \in[0,1]
$$

where $\rho_{d}=\rho_{\text {martensite }}$ is partial mass density of the daughter phase, $\rho=\rho_{d}+\rho_{p}$ is total mass density, and $\rho_{p}=\rho_{\text {ferrite }}$ is partial mass density of the parent phase (i.e., the ferrite phase);

(iii) the total infinitesimal strain $\epsilon$ can be written as follows:

$$
\begin{aligned}
& \epsilon=\epsilon^{\mathrm{e}}+\epsilon^{\mathrm{th}}+\epsilon^{\mathrm{pc}}+\epsilon^{\mathrm{tr}}+\epsilon^{\mathrm{pz}} \\
& \text { with } \epsilon^{\mathrm{th}}=\alpha\left(T-T_{0}\right), \epsilon^{\mathrm{tr}}=-\frac{z}{\mathcal{\kappa}},
\end{aligned}
$$

where $\epsilon^{\mathrm{e}}, \epsilon^{\mathrm{th}}, \epsilon^{\mathrm{pc}}$ denote the elastic, thermal, classical (visco-) plasticity strains, respectively, $\epsilon^{\text {tr }}$ is the transformation strain associated with the volume variation, $\epsilon^{\mathrm{pz}}$ is TRIP-like strain, $T_{0}$ is initial temperature, $\alpha>0$ is the thermal expansion coefficient, and $\kappa>0$ is a material parameter characterizing the change in the density occurring during phase transformation. Note that the solid-solid phase transformation under consideration here induces a volume change, or more specifically, a decrease in the volume, that is, $\epsilon^{\operatorname{tr}}=-z / \kappa<0$, since the density mass of ferrite (the initial material of the rail before phase transformation) $\rho_{\text {ferrite }} \approx 7700 \mathrm{~kg} \cdot \mathrm{m}^{-3}$, and that of martensite (material after phase transformation) $\rho_{\text {martensite }} \approx 7800 \mathrm{~kg} \cdot \mathrm{m}^{-3}$.

The material in question is initially nontransformed $\left(z_{0}=0, \epsilon_{0}^{\mathrm{tr}}=0\right.$ and $\left.\epsilon_{0}^{\mathrm{pz}}=0\right)$ and nonplasticized $\left(v_{0}=0\right.$, and $\epsilon_{0}^{\mathrm{pc}}=0$ where $v$ is the isotropic hardening variable associated with classical plasticity) at the temperature $T_{0}$, and the Helmholtz free energy potential per unit mass is written as:

$$
\begin{aligned}
\psi\left(T, \epsilon, \epsilon^{\mathrm{pc}}, \epsilon^{\mathrm{pz}}, v, z\right) \\
=-\frac{C_{\epsilon}}{2 T_{0}}\left(T-T_{0}\right)^{2}-\frac{E}{\rho_{0}} \alpha\left(T-T_{0}\right) \epsilon \\
+\frac{E}{2 \rho_{0}}\left[\epsilon-\epsilon^{\mathrm{pc}}-\epsilon^{\mathrm{tr}}-\epsilon^{\mathrm{pz}}\right]^{2}+\frac{1}{2 \rho_{0}} h v^{2} \\
+\frac{\delta}{\rho_{0} \kappa}\left[\frac{T}{2} z^{2}-\left(T-T_{i}^{z}\right) z\right] \\
+I_{[0,1]}(z)+\psi_{0},
\end{aligned}
$$

where $C_{\epsilon}>0$ is the specific heat capacity, $E$ is Young's modulus, $T_{i}^{z}$ is the solid-solid phase transformation temperature, $h>0$ is a material parameter characterizing the linear isotropic hardening associated with classical plasticity, $\delta \geq 0$ is a material parameter associated with the latent heat of the phase transformation, $I_{[0,1]}(z)$ is the indicator function $\left(I_{[0,1]}(z)=0\right.$ when $z \in[0,1]$ and $I_{[0,1]}(z)=+\infty$ otherwise $)$ and $\rho_{0}$ and $\psi_{0}$ are the initial density and the initial Helmholtz free energy of the material per unit mass, respectively. In a first approximation, the thermoelastic parameters, $C_{\epsilon}, E$, and $\alpha$, are not assumed to depend on the temperature and are taken to be identical in the both phases. Equation (3) is strongly inspired by SMAs model with a $1-\mathrm{D}-\mathrm{Helmholtz}$ free energy potential developed by [10] where the thermomechanical coupling process considered here is extended to include an irreversible solid-solid phase transformation. Note that in (3), the first two terms correspond to the thermal leaks (irreversible part), the third one corresponds to the free energy part at constant temperature which may be immediately recoverable by an elastic unloading (reversible part), and the next two terms correspond to the hardening associated with the classical plasticity (isotropic hardening) and the latent heat of the phase transformation, respectively.

Neglecting the viscoelastic effects and noting that the specific entropy $S$ is a state function, the state equations based on the above representation of the Helmholtz energy (3) can be written as:

$$
S=-\frac{\partial \psi}{\partial T}, \quad \sigma=\rho \frac{\partial \psi}{\partial \epsilon}, \quad A^{m}=-\rho \frac{\partial \psi}{\partial m}
$$

where $\sigma$ is the Cauchy stress tensor, and $A^{m}$ denote the "thermodynamic forces" associated with the variables $m=\left(\epsilon^{\mathrm{pc}}, \epsilon^{\mathrm{tr}}, \epsilon^{\mathrm{pz}}, v, z\right)$.

Using (3)-(4) and assuming that $\rho \simeq \rho_{0}$, the Cauchy stress tensor $\sigma$ is:

$$
\sigma=E\left(\epsilon-\alpha\left(T-T_{0}\right)-\epsilon^{\mathrm{pc}}-\epsilon^{\mathrm{tr}}-\epsilon^{\mathrm{pz}}\right) .
$$

The local expression for the First Principle of Thermodynamics can be written (see e.g., [11]) as:

$$
\rho \dot{e}=\sigma \dot{\epsilon}-\operatorname{div}(\mathbf{q}) \Longleftrightarrow \rho T \dot{S}+\operatorname{div}(\mathbf{q})=\sigma \dot{\epsilon}-\rho S \dot{T}-\rho \dot{\psi},
$$

where $e=\psi+T S$ denotes the internal energy potential per unit mass, $\mathbf{q}$ is the heat flux vector; the remote heat production rate has been omitted here.

The Second Principle of Thermodynamics must be checked, whatever the local thermomechanical state $\mathcal{S}_{\mathrm{tm}}=\left(T, \epsilon, \epsilon^{\mathrm{pc}}, \epsilon^{\mathrm{tr}}, \epsilon^{\mathrm{pz}}, v, z\right)$ of the material, whatever the evolution $(\dot{T}, \dot{\epsilon})$ locally undergone by the material, and whatever the temperature gradient $\nabla T$ acting locally on the material (see [10]) as follows:

$$
-\frac{1}{T} \mathbf{q} \cdot \nabla T+\rho T \dot{S}+\operatorname{div}(\mathbf{q}) \geq 0 \quad \forall \mathcal{S}_{\mathrm{tm}}, \forall(\dot{T}, \dot{\epsilon}), \forall \nabla T
$$


Adopting Fourier's law $(\mathbf{q}=-k \nabla T$, where $k>0$ denotes the thermal conductivity), and combining (3)-(4) and (7), the Second Principle of Thermodynamics must satisfy:

$$
A^{m} \dot{m} \geq 0 \quad \forall \mathcal{S}_{\mathrm{tm}}, \forall(\dot{T}, \dot{\epsilon}), \forall \nabla T
$$

The laws of evolution of the internal state variables are.

(i) In the irreversible solid-solid phase transformation (TST) process:

$$
\dot{\epsilon}^{\mathrm{pz}}=\dot{p} \frac{\sigma}{|\sigma|}, \quad \dot{\epsilon}^{\mathrm{tr}}=-\frac{\dot{z}}{\kappa}, \quad \dot{z}=\kappa \dot{p}
$$

with

$$
\dot{p}=\langle 1-z\rangle \frac{\left\langle f^{\mathrm{pz}}(\sigma, z ; T)\right\rangle}{\bar{\sigma}_{0} \eta} H\left(|\sigma|+\delta\left[(1-z) T-T_{i}^{z}\right]\right) \geq 0
$$

and

$$
f^{\mathrm{pz}}(\sigma, z ; T)=|\sigma|-\left(\chi \bar{\sigma}_{0} \exp \left(\frac{T_{0}-T}{T_{0}}\right)+\beta \frac{z}{\kappa}\right)
$$

where $\bar{\sigma}_{0}$ denotes the yield strength of the softest phase (parent phase) in the TST when $T=T_{0}, \chi$ and $\beta$ are a material parameter associated with TST's yield strength and the hardening process, respectively, $|\cdot|$ is the absolute value function $(|x|=x$ when $x \geq 0$ and $|x|=-x$ when $x<0)$, $\langle\cdot\rangle$ denotes the Macaulay brackets $(\langle x\rangle=x$ when $x \geq 0$ and $\langle x\rangle=0$ when $x<0), \eta$ is the characteristic time of the viscous effects associated with the TST process, and $H(\cdot)$ denotes the Heaviside step function $(H(x)=1$ when $x \geq 0$ and $H(x)=0$ when $x<0$ ).

It must be stressed that: (i) TSTs (or WELs), which correspond to the ferrite-pearlite $\rightarrow$ martensite transformations, do not fit the framework of conventional metallurgical transformations. According to these metallurgical theories, the white layer (martensite) may result from the martensitic transformation (high velocity cooling) after a sufficiently large temperature increase to produce an austenitic transformation. Dang Van et al. [12] have established that in the case of a steadily moving train, the temperature will increase at the surface of the rails by $150^{\circ} \mathrm{C}$ or so at most. Therefore, this temperature increase alone, which is caused by friction involved in the contact, is obviously too small to generate solid-solid phase transformations. The assumption adopted here is therefore that the thermomechanical coupling, where the thermal stresses superimposed on the purely mechanical (isothermal) stresses, can be taken to generate TSTs. (ii) Equation (11) means that the irreversible solid-solid phase transformation is activated by the stress $\sigma$ which acts as "driving force," and accelerated by temperature $T$ (see [9] for more details). TST occurs at stress levels which trigger of dislocation motions due to the strain incompatibilities between the ferrite-pearlite and martensite phases. The triggering of these dislocation motions is only facilitated by the increase in the temperature. In agreement with (i), (10) reflects the fact that the validity field of the law of evolution governing the TST process requires the presence of both the stress and the temperature.

(ii) In the classical plasticity process:

$$
\dot{\epsilon}^{\mathrm{pc}}=\dot{v} \frac{\sigma}{|\sigma|} \quad \text { with } \dot{v}=\frac{\left\langle f^{\mathrm{pc}}(\sigma, v)\right\rangle}{\xi \sigma_{y}(z)} \geq 0,
$$

with

$$
f^{\mathrm{pc}}(\sigma, v)=|\sigma|-\left(\sigma_{y}(z) \exp \left(\frac{T_{0}-T}{T_{0}}\right)+h v\right)
$$

and

$$
\sigma_{y}(z)=(1-z) \bar{\sigma}_{0}+z \bar{\sigma}_{1}
$$

where $\sigma_{y}(z)$ denotes the classical yield strength of the material when $T=T_{0}, \bar{\sigma}_{1}$ is the yield strength of the hardest phase (daughter phase) and $\xi$ is the characteristic time of the viscous effects associated with the classical plasticity process. Note that when $z=0$ (resp., $z=1$ ), the yield strength $\sigma_{y}(z)$ is only composed to one phase, that is, the softest phase (resp., the hardest phase) and it consists of two phases when $z \in] 0,1[$.

Combining (3), (4), and (6) and Fourier's law, the heat equation is given by:

$$
\begin{aligned}
& \rho C_{\epsilon} \frac{T}{T_{0}} \dot{T}-k \Delta T \\
& \quad=-E \alpha T \dot{\epsilon}+\sigma \dot{\epsilon}^{\mathrm{pc}}-h v \dot{v}+\sigma\left(\dot{\epsilon}^{\mathrm{tr}}+\dot{\epsilon}^{\mathrm{pz}}\right)-T_{i}^{z} \frac{\delta}{\kappa} \dot{z},
\end{aligned}
$$

where $\Delta$ denotes the Laplacian operator. The quantities on the right-hand side of (15) are the "source terms" corresponding to the thermoelasticity, the classical plasticity, and the irreversible solid-solid phase transformation, respectively.

It is worth noting the thermodynamic consistency of this model [9] is checked since the laws of evolution of the internal state variables verify systematically (8).

\section{Numerical Example}

Preliminary Remark. The case of the structure studied in this section is not representative of the complex processes that could be expected between wheel and rail, such as nonlinear contact and friction. This one-dimensional problem was simply intended to show that the thermomechanical coupling can generate the initiation and development of TSTs in the immediate vicinity where a sufficient thermomechanical load level is applied on the structure. Therefore, it leads on naturally to first the step to the setting up of a TSTs benchmark.

This example will be addressed in order to assess the thermomechanical coupling occurring in the TSTs using the 
approach presented in Section 2, subject to the assumptions stated therein. Let us consider a solid occupying a onedimensional space $\Omega \in[0, L] \subset \mathfrak{R}$, measuring $10 \mathrm{~mm}$ of length, that is, a solid bar where the material considered here is made of steel. On the left end of the bar, a thermomechanical loading $\left\{\sigma_{0}^{g}(t), T_{0}^{g}(t)\right\}$, which progresses linearly in conjunction with time $t$, is applied whereas both zero displacement $\left(u_{L}^{g}(t)=0\right)$ and a set temperature $\left(T_{L}^{g}(t)=T_{0}\right)$ are imposed constant with time on the right end of the bar, that is, $u_{L}^{g}(t)=0, \forall t$ and $T_{L}^{g}(t)=T_{0}, \forall t$ (see Figure 1 ). The mechanical component of the loading is a compression which decreases from zero to $-300 \mathrm{MPa}$ in $0 \leq t \leq 0.1 \mathrm{~s}$ and its thermal component, and it increases from $300 \mathrm{~K}$ to $450 \mathrm{~K}$ in the same time. Bearing in mind that the one-dimensional problem studied cannot take into account both the nonuniformity of normal and shear stresses in contact area including the temperature increase caused by friction, the maximum values chosen to describe the thermomechanical loading would seem to be realistic enough at a contact point under moderate load for pressure and temperature in increase. Furthermore, in a first approximation, the lateral surfaces of the bar is assumed to be adiabatic thereby both the temperature gradient $\nabla T$ and heat flux $\mathbf{q}$ are onedimensional (here, $x$-direction), that is, $\nabla T=(\partial T / \partial x) \mathbf{e}_{x}, \forall t$ and $\mathbf{q}=-k(\partial T / \partial x) \mathbf{e}_{x}, \forall t$ (where $\mathbf{e}_{x}$ is a unit vector).

Assuming that the convective terms of particular derivative are zero, (15) can be written in the following form:

$$
\begin{aligned}
\rho C_{\epsilon} \frac{T}{T_{0}} & \frac{\partial T}{\partial t}-k \frac{\partial^{2} T}{\partial x^{2}} \\
= & -E \alpha T \frac{\partial \epsilon}{\partial t}-h v \frac{\partial v}{\partial t}-T_{i}^{z} \frac{\delta}{\kappa} \frac{\partial z}{\partial t} \\
& +\sigma\left(\frac{\partial \epsilon^{\mathrm{tr}}}{\partial t}+\frac{\partial \epsilon^{\mathrm{pz}}}{\partial t}+\frac{\partial \epsilon^{\mathrm{pc}}}{\partial t}\right) \quad \text { in } \Omega,
\end{aligned}
$$

where $\partial(\cdot) / \partial t$ and $\partial^{2}(\cdot) / \partial x^{2}$ denote the first and second partial derivatives with respect to $t$ and $x$ (temporal and spatial quantities).

Neglecting the effects of both the inertia and gravity, the local expression of quasi-static equilibrium reduces to:

$$
\frac{\partial \sigma}{\partial x}=0 \quad \text { in } \Omega
$$

In line with (17), the stress field $\sigma(x, t)$ is uniform, that is, $\sigma(x, t)=\sigma_{0}^{g}(t), \forall x \in[0, L]$. The geometry involved and the boundary conditions are summarized in Figure 1. The constitutive thermomechanical parameters used are: $C_{\epsilon}=450 \mathrm{~J} \cdot \mathrm{kg}^{-1} \cdot \mathrm{K}^{-1} ; E=2 \times 10^{5} \mathrm{MPa} ; \rho=78 \times 10^{-7}$ $\mathrm{kg} \cdot \mathrm{mm}^{-3} ; \alpha=12 \times 10^{-6} \mathrm{~K}^{-1} ; \kappa=10^{2} ; \bar{\sigma}_{0}=380 \mathrm{MPa} ;$ $\bar{\sigma}_{1}=465 \mathrm{MPa} ; k=5 \times 10^{-2} \mathrm{~W} \cdot \mathrm{mm}^{-1} \cdot \mathrm{K}^{-1} ; \eta=10^{2} \mathrm{~s} ;$ $\xi=2 \times 10^{2} \mathrm{~s} ; \beta=6 \times 10^{3} \mathrm{MPa} ; h=2 \times 10^{4} \mathrm{MPa} ; \chi=4 / 5 ;$ $T_{i}^{z}=1000 \mathrm{~K} ; \delta=2.5 \times 10^{-10} \mathrm{~J} \cdot \mathrm{mm}^{-3} \cdot \mathrm{K}^{-1}$. Note that $C_{\epsilon}$, $k, E$, and $\alpha$ are classical thermoelastic parameters associated with a ferritic or martensitic steel, which are considered here constants in temperature range studied. As pointed out in Section 2 , the material parameter $\kappa$ characterizing the change in the density occurring during phase transformation (where density mass of ferrite $\rho_{\text {ferrite }} \approx 7700 \mathrm{~kg} \cdot \mathrm{m}^{-3}$ and martensite $\rho_{\text {martensite }} \approx 7800 \mathrm{~kg} \cdot \mathrm{m}^{-3}$ ). Therefore, based on these experimental data, the transformation ferrite $\rightarrow$ martensite is accompanied by a volume contraction (for a constant mass given) to be taken into account, that is,

$$
\rho_{\text {martensite }} \cong\left(1+2 \times 10^{-2}\right) \rho_{\text {ferrite }}
$$

On the other hand, the equation of conservation of mass (under small perturbations assumption) can be written as:

$$
\rho \simeq \rho_{0}(1-\operatorname{tr}(\boldsymbol{\epsilon})) \Longleftrightarrow \rho \simeq \rho_{0}(1-\epsilon) \quad \text { in } \Omega .
$$

Combining (5), (9), and (12) and considering both a return to thermal and mechanical equilibrium (i.e., $T=T_{0}$ and $\sigma=0$ ), we can write that:

$$
\epsilon=\epsilon^{\mathrm{pc}}+\epsilon^{\mathrm{tr}}+\epsilon^{\mathrm{pz}}
$$

Assuming both that $\left|\epsilon^{\mathrm{pc}}\right| \lll\left|\epsilon^{\mathrm{tr}}\right|$ and $\left|\epsilon^{\mathrm{pc}}\right| \lll\left|\epsilon^{\mathrm{pz}}\right|$ and using (9) (here for a mechanical evolution $\dot{\sigma}<0$ and then a return to the mechanical state $\sigma=0),(20)$ can be rewritten:

$$
\epsilon=-2 \frac{\mathrm{Z}}{\kappa}
$$

In the case of full phase transformation $\left(z=z_{\max }=1\right),(21)$ can be reduced to:

$$
-\epsilon=\frac{2}{\kappa} .
$$

Combining (19) and (22), the expression of (18) can be put in the following form:

$$
\rho_{\text {martensite }} \cong\left(1+2 \kappa^{-1}\right) \rho_{\text {ferrite }} .
$$

From (18), we can deduce that $\kappa=10^{2}$. Note also that the characteristic times of the viscous effects associated with the TRIP-like process $\eta$ and the classical plasticity $\xi$ are rather low which means that the viscous effects are also small (when $\eta \rightarrow 0$ and $\xi \rightarrow 0$, these are nonexistent). The reader is invited to refer to [9] the physical interpretations associated with other parameters including their chosen set value.

The results of the numerical simulations are presented in Figures 2, 3, 4, and 5. They show that due to both the nonstationary heat equation with source terms (16) and quasistatic equilibrium (17) combined with the thermomechanical loading (see Figure 1), a uniform $\sigma$-field (see Figure 2(a)) and a non-uniform temperature distribution strongly localized (near where the load is applied, see Figure 2(b)) are obtained throughout of the bar. These source terms present in the heat equation will provide a maximum temperature increase of a few degrees (around $15 \mathrm{~K}$ ) which occurs near the left end of the bar (at time $t_{D}$ ), whereas no such increase occurred elsewhere. The increase of about $10 \mathrm{~K}$, which can be observed in the close vicinity of the point where the thermomechanical loading is applied (in the same time), is due only to the 


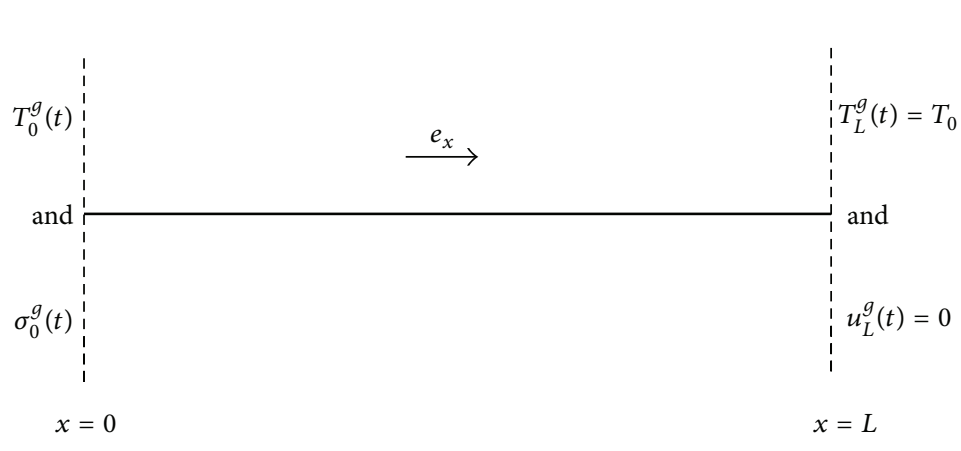

(a)

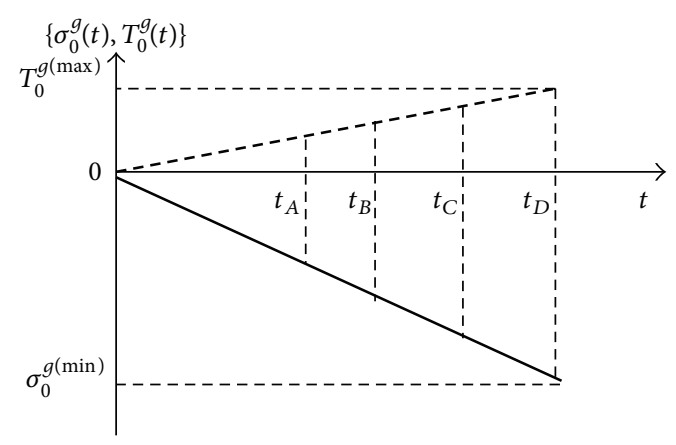

(b)

FIGURE 1: (a) Numerical example of TSTs: bar subjected to a thermomechanical loading (where $L=10 \mathrm{~mm}$ ). (b) Boundary conditions (where $T_{0}=300 \mathrm{~K}$ ): the loading function $\sigma_{0}^{g}(t)$ (resp., $T_{0}^{g}(t)$ ) is represented in solid lines with $\sigma_{0}^{g(\min )}=-300 \mathrm{MPa}$ at time $t_{D}=10^{-1} \mathrm{~s}(\mathrm{resp}$., dashed lines with $\left.T_{0}^{g(\max )}=450 \mathrm{~K}\right)$. Note that $t_{A}=7.3 \times 10^{-2} \mathrm{~s}$ with $\sigma_{0}^{g}\left(t_{A}\right)=-220 \mathrm{MPa}$ and $T_{0}^{g}\left(t_{A}\right)=409.5 \mathrm{~K} ; t_{B}=7.5 \times 10^{-2} \mathrm{~s}$ with $\sigma_{0}^{g}\left(t_{B}\right)=-224 \mathrm{MPa}$ and $T_{0}^{g}\left(t_{B}\right)=412 \mathrm{~K} ; t_{C}=9.4 \times 10^{-2} \mathrm{~s}$ with $\sigma_{0}^{g}\left(t_{C}\right)=-283 \mathrm{MPa}$ and $T_{0}^{g}\left(t_{C}\right)=441 \mathrm{~K}$.

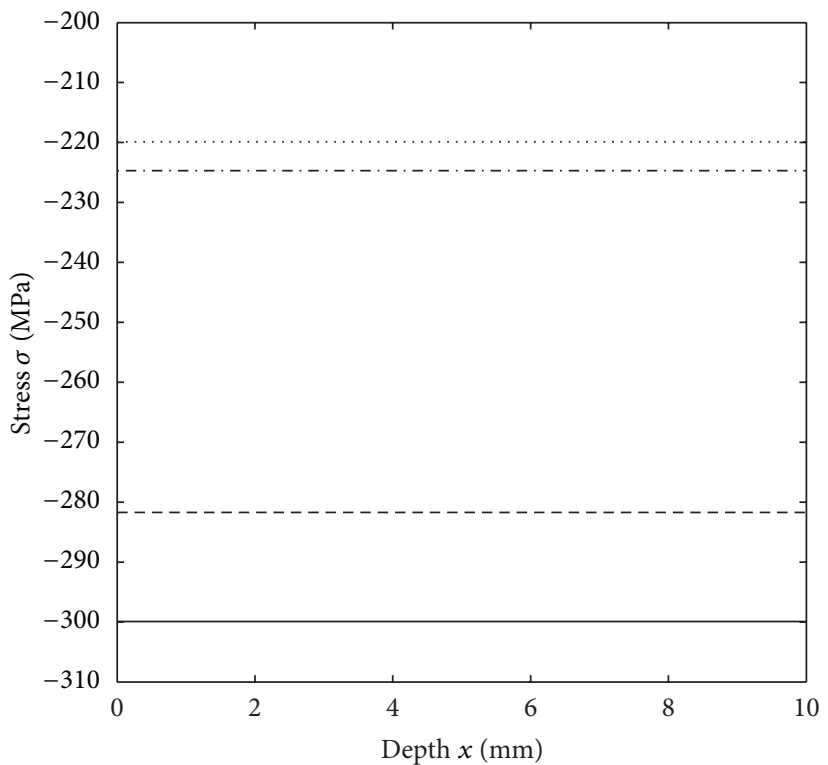

(a)

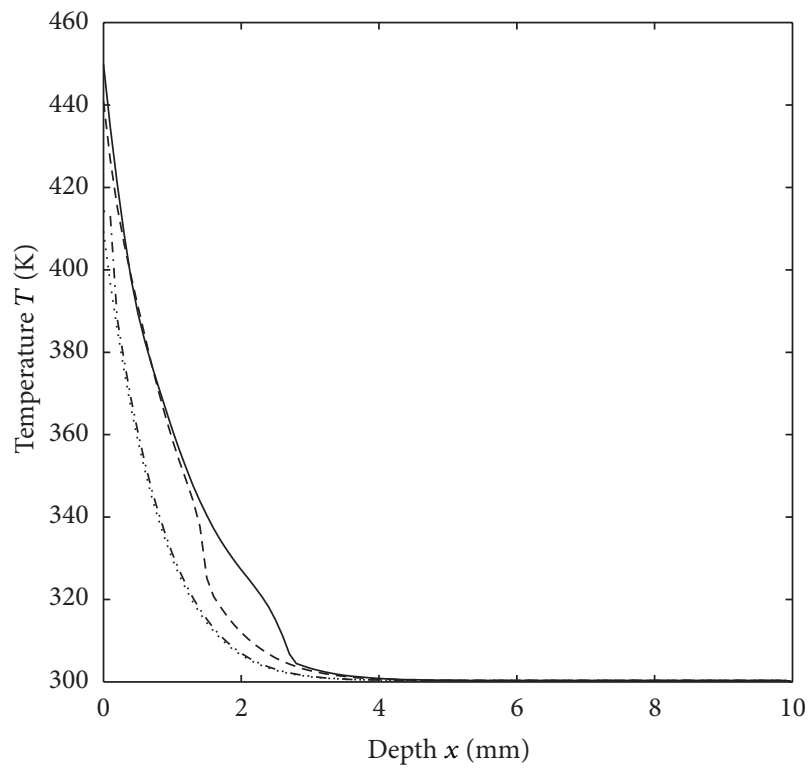

(b)

Figure 2: (a) $\sigma$-field in the depth of the bar at time $t_{A}$ (dotted line), $t_{B}$ (dashed-dotted line), $t_{C}$ (dashed line), and $t_{D}$ (solid line). (b) Distribution of temperature $T$ in the depth of the bar at time $t_{A}$ (dotted line), $t_{B}$ (dashed-dotted line), $t_{C}$ (dashed line), and $t_{D}$ (solid line).

thermoelastic coupling. These results indicate that a "new" phase $(z \neq 0)$ develops in the immediate vicinity of the point on the bar where the thermomechanical loading is applied: from $x=0$ to about $0.2 \mathrm{~mm}$ at time $t_{B}, 1.6 \mathrm{~mm}$ at time $t_{C}$, and $2.8 \mathrm{~mm}$ in depth at time $t_{D}$ (see Figure 3(a)). Figure 3(b) brings out the distribution of the TRIP-like strain $\epsilon^{p z}$ in the depth of the bar at time $t=\left\{t_{B}, t_{C}, t_{D}\right\}$. Note that at time $t_{A}, z=\epsilon^{\mathrm{pz}}=0, \forall x$. A full phase transformation $\left(z=z_{\max }=1\right)$ develops up to about $0.1 \mathrm{~mm}$ at time $t_{D}$ (see Figure 4(a)), where there is only one phase, the daughter phase (martensite). At depths of $0.1<x<2.8 \mathrm{~mm}$, the partial transformation $(z<1)$ persists, that is, the material is a mixture between the parent phase (ferrite-pearlite) and the daughter phase (martensite). Distribution of transformation strain $\epsilon^{\text {tr }}$ in total bar length is also depicted in the same time. Figure 4(b) shows the distribution of the TRIP-like and classical plasticity strain in the depth of the bar at time $t_{D}=10^{-1} \mathrm{~s}$. Near the left end of the bar, the TRIP-like strain $\epsilon^{p z}$ predominates, reaching with a extremum value of $-10^{-2}$ at time $t_{D}=10^{-1} \mathrm{~s}$, whereas the strain associated with classical plasticity amounted to $\epsilon^{\mathrm{pc}} \approx-0.0085 \times 10^{-6}$ at the same time. It can be observed that at the point $x=L / 100$ (Figure 5): (i) on the loading path $0 \rightarrow A$ (where $\left.t \in\left[0, t_{A}\right]\right)$, the response of the material is purely thermoelastic (i.e., $z=\epsilon^{\mathrm{tr}}=\epsilon^{\mathrm{pz}}=\epsilon^{\mathrm{pc}}=0$ ). The thermal effects gives a non-linear stress-strain curve; (ii) on the loading path $A \rightarrow C$ (where $t \in\left[t_{A}, t_{C}\right]$ ), thermo-elasto-viscoplastic behavior occurs due to the solid-solid phase transformation 


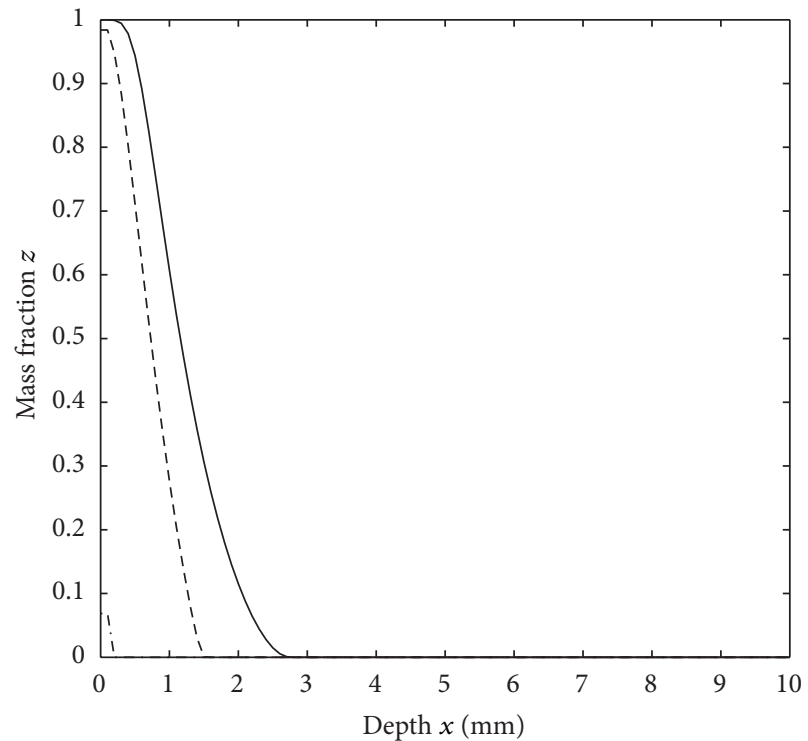

(a)

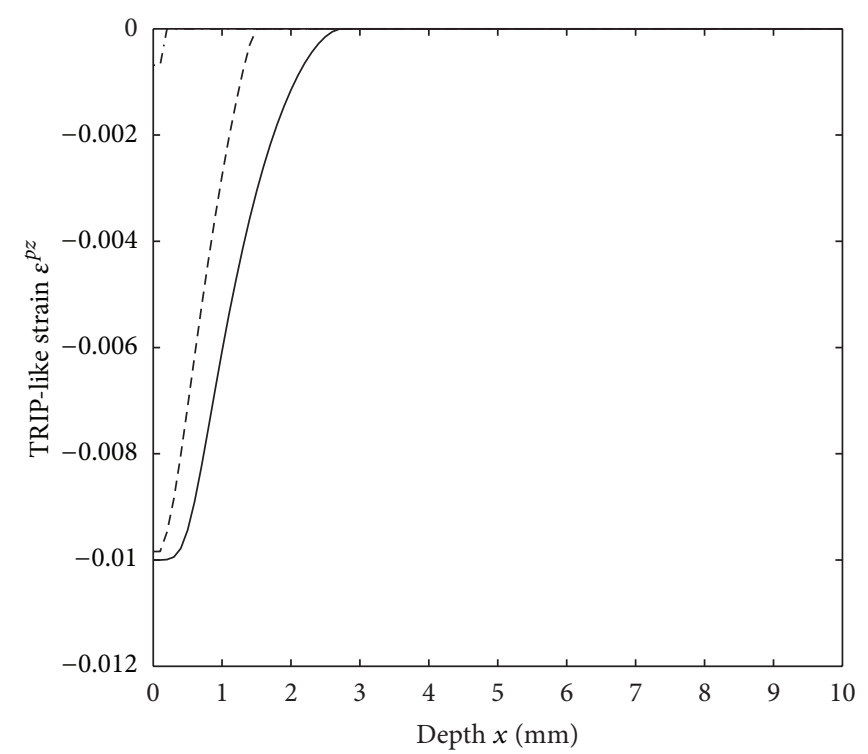

(b)

Figure 3: (a) $z$-field in the depth of the bar at time $t_{B}$ (dashed-dotted line), $t_{C}$ (dashed line), and $t_{D}$ (solid line). (b) Distribution of the TRIP-like strain $\epsilon^{\mathrm{pz}}$ in the depth of the bar at time $t_{B}$ (dashed-dotted line), $t_{C}$ (dashed line), and $t_{D}$ (solid line) and at time $t_{A}, z=\epsilon^{\mathrm{pz}}=0, \forall x$.

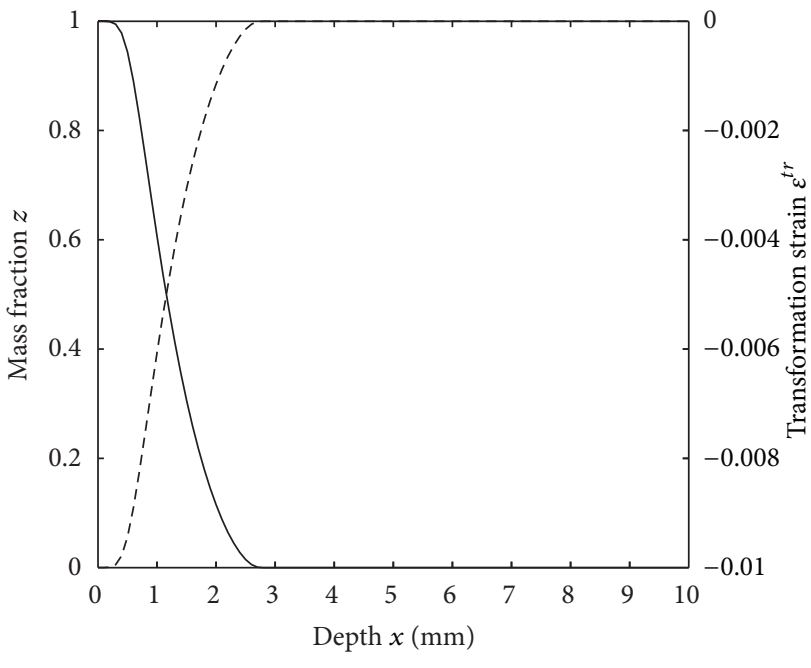

(a)

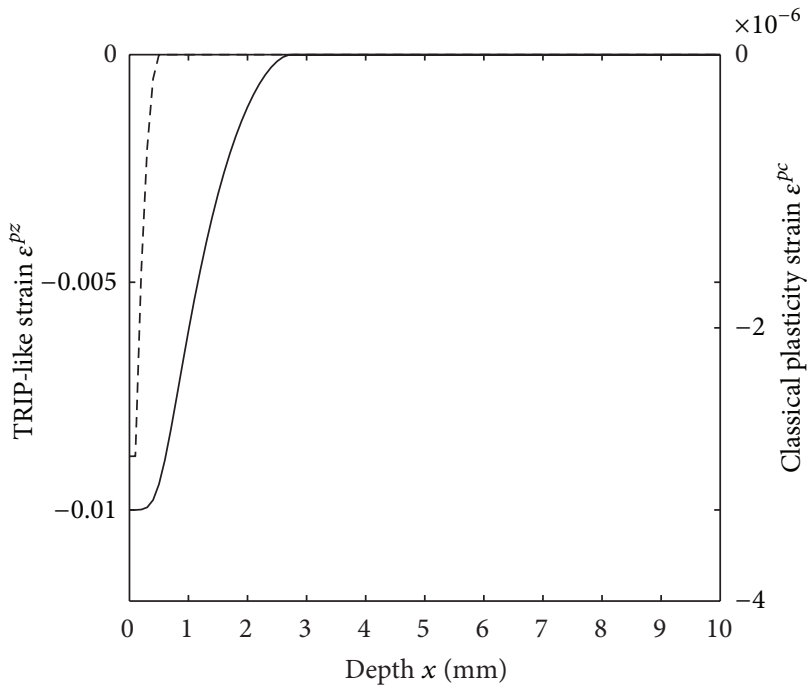

(b)

FIGURE 4: (a) Distribution of mass fraction $z$ (solid line) and transformation strain $\epsilon^{\text {tr }}$ (dashed line) in the depth of the bar at time $t=t_{D}$. (b) Distribution of the TRIP-like strain, $\epsilon^{\mathrm{pz}}$ (solid line), and the classical plasticity strain, $\epsilon^{\mathrm{pc}}$ (dashed line), in the depth of the bar at time $t=t_{D}$.

and classical plasticity (i.e., $z \neq 0, \epsilon^{\mathrm{tr}} \neq 0, \epsilon^{\mathrm{pz}} \neq 0, \epsilon^{\mathrm{pc}} \neq 0$ ); (iii) on the loading path $C \rightarrow D$ (where $t \in\left[t_{C}, t_{D}\right]$ ), a return to the thermoelastic response of the material (i.e., $\dot{z}=\dot{\epsilon}^{\text {tr }}=\dot{\epsilon}^{\mathrm{pz}}=\dot{\epsilon}^{\mathrm{pc}}=0$ ) occurs.

The results presented in Section 3 are in line with the experimental available data on TSTs [1], since a full layer transformed is strongly localized and achieved a realistic TST thickness, that is, at a maximum depth of around $100 \mu \mathrm{m}$ (see Section 1). The thermomechanical model proposed here can describe both the initiation and development of TSTs at the surface and in the immediate vicinity where the thermomechanical loading was applied. Therefore, this model will be examined on the wheel/rail contact where both the nonlinear contact and friction can take place.

\section{Conclusion}

The thermodynamically consistent model presented in this study can be used to simulate the initiation and development 


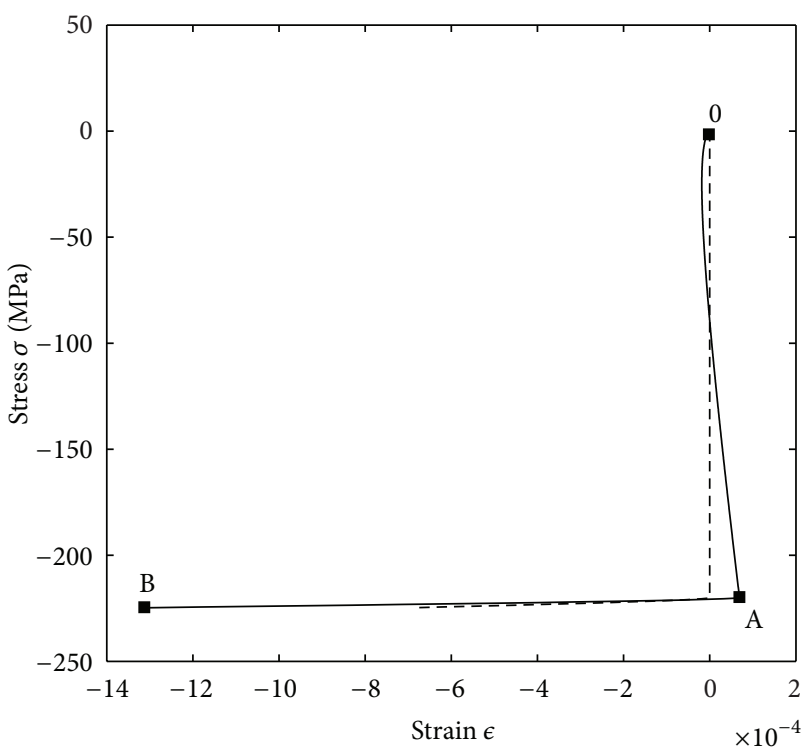

(a)

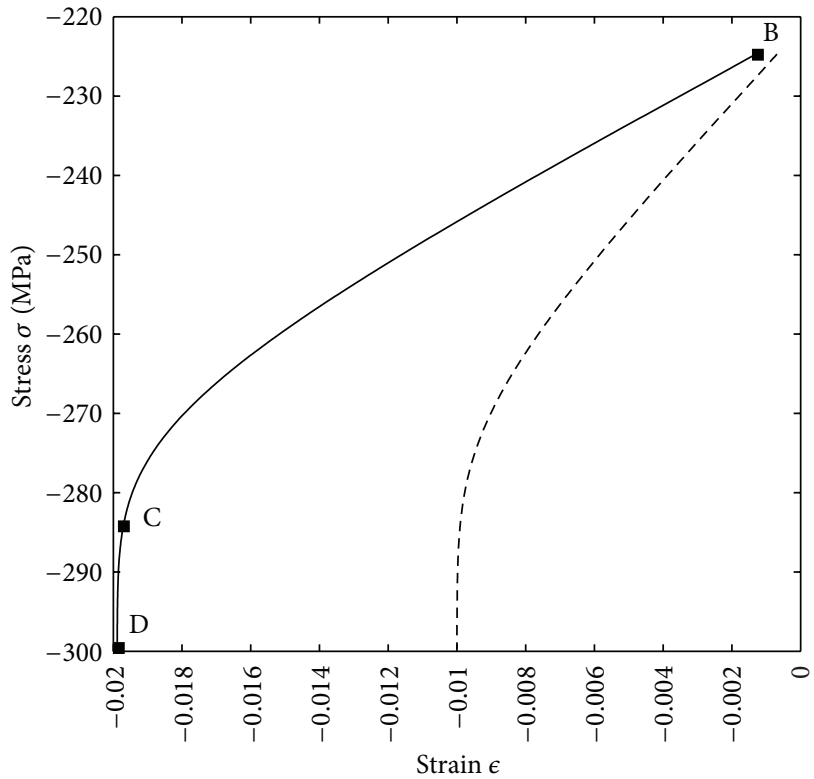

(b)

Figure 5: Stress/strain curve (solid line) and stress/TRIP-like strain curve (dashed line) in $x=L / 100$ at time $t \in\left[0, t_{B}\right](\mathrm{a})$ and $t \in\left[t_{B}, t_{D}\right]$ (b).

of Tribological Surface Transformations (TSTs). The results obtained here show that the thermomechanical coupling can generate a thin layer of fully irreversible solid-solid phase transformation in the immediate vicinity of the point on the bar where the thermomechanical loading is applied. These results also highlight that the layer thickness transformed is in line with the experimental available data on TSTs since a depth of around one hundred micrometers has been reached. In the future studies, it is proposed to test the present thermomechanical model in more realistic numerical simulations involving issues relatied to wheel/rail contact problems.

\section{Acknowledgment}

The author is indebted to Professor Thierry Désoyer and Professor Frédéric Lebon for the valuable discussions.

\section{References}

[1] Österle, Rooch, Pyzalla, and Wang, "Investigation of white etching layers on rails by optical microscopy, electron microscopy, $\mathrm{X}$-ray and synchroton X-ray diffraction," Materials Science and Engineering A, vol. 303, no. 1-2, pp. 150-157, 2001.

[2] G. Baumann, H. J. Fecht, and S. Liebelt, "Formation of whiteetching layers on rail treads," Wear, vol. 191, no. 1-2, pp. 133-140, 1996.

[3] G. W. Greenwood and R. H. Johnson, "The deformation of metals under small stresses during phase transformations," Proceedings of the Royal Society of London A, vol. 293, pp. 403422, 1965.

[4] Leblond, Devaux, and Devaux, "Mathematical modelling of transformation plasticity in steels I: case of ideal-plastic phases," International Journal of Plasticity, vol. 5, no. 6, pp. 551-572, 1989.
[5] J. B. Leblond, J. Devaux, and J. C. Devaux, "Mathematical modelling of transformation plasticity in steels II: coupling with strain hardening phenomena," International Journal of Plasticity, vol. 5, no. 6, pp. 573-591, 1989.

[6] J.-C. Videau, G. Cailletaud, and A. Pineau, "Experimental study of the transformation induced plasticity in a Cr-Ni-Mo-Al-Ti steel," Journal de Physique, vol. 6, Supplément au Journal de Physique III, no. 1, pp. 465-474, 1996.

[7] L. Taleb and F. Sidoroff, "A micromechanical modeling of the Greenwood-Johnson mechanism in transformation induced plasticity," International Journal of Plasticity, vol. 19, no. 10, pp. 1821-1842, 2003.

[8] L. Taleb and S. Petit, "New investigations on transformation induced plasticity and its interaction with classical plasticity," International Journal of Plasticity, vol. 22, no. 1, pp. 110-130, 2006.

[9] G. Antoni, Transformations Tribologiques de Surface: Une Approche Thermo-Mécanique [Ph.D. thesis], Université de Provence, Marseille, France, 2010.

[10] A. Chrysochoos, C. Licht, and R. Peyroux, "A one-dimensional thermomechanical modeling of phase change front propagation in a SMA monocrystal," Comptes Rendus Mécanique, vol. 331, no. 18, pp. 25-32, 2003.

[11] J. Garrigues, Fondements de la Mécanique des Milieux Continus, Hermes Science Publications, Paris, France, 2007.

[12] K. Dang Van, M. H. Maitournam, and B. Prasil, "Elastoplastic analysis of repeated moving contact application to railways damage phenomena," Wear, vol. 196, no. 1-2, pp. 77-81, 1996. 

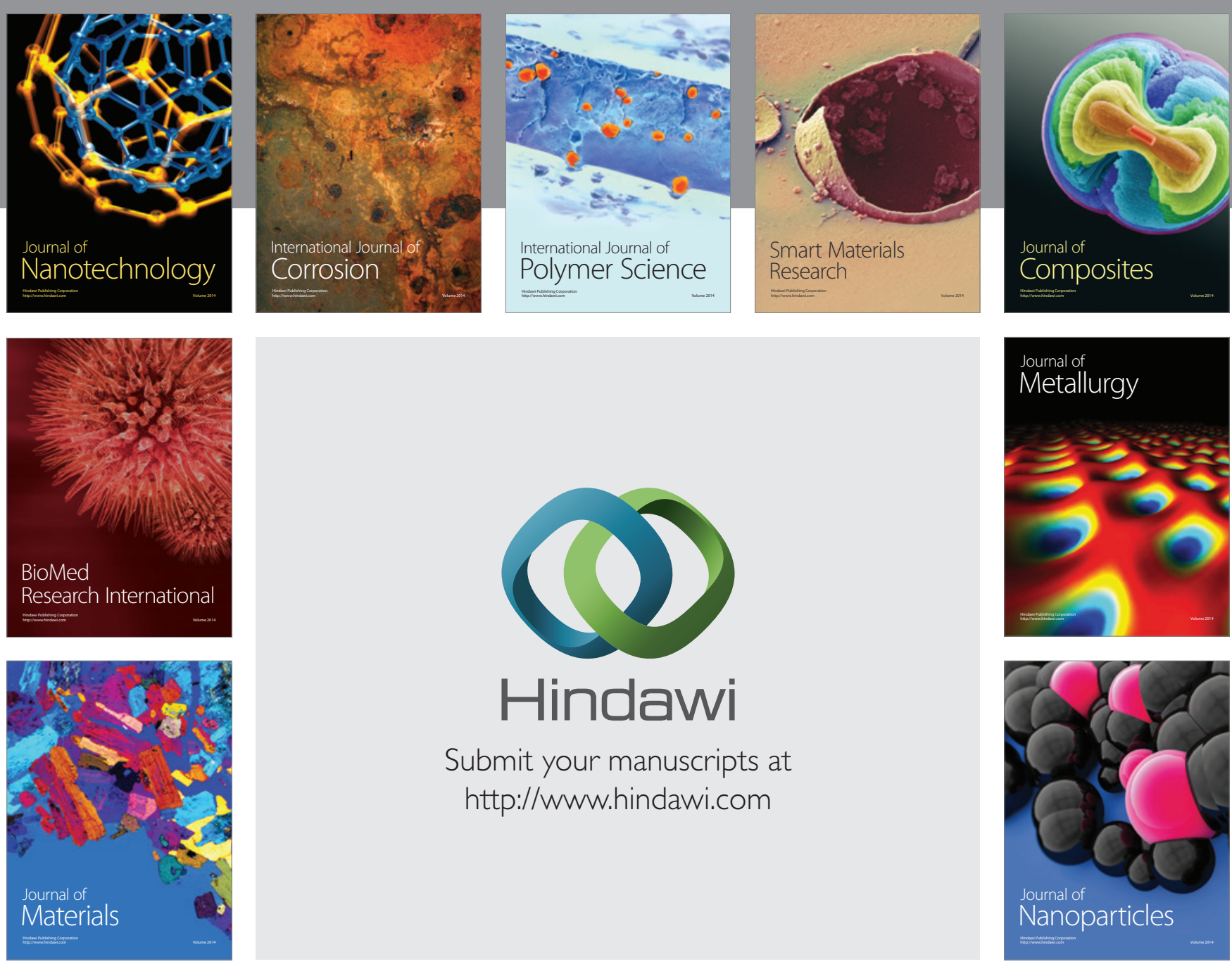

Submit your manuscripts at http://www.hindawi.com
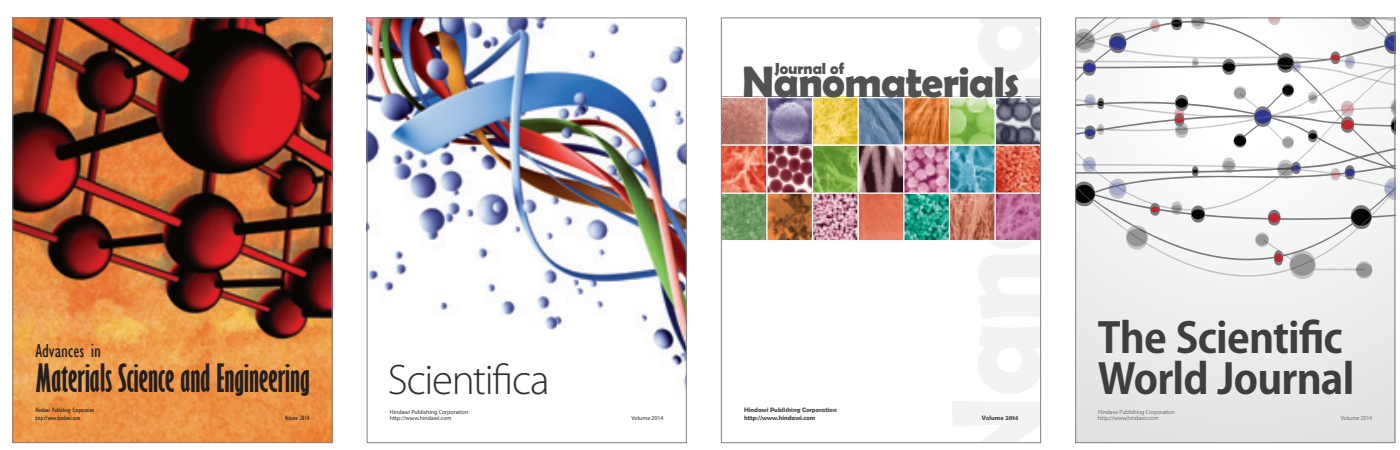

\section{The Scientific World Journal}
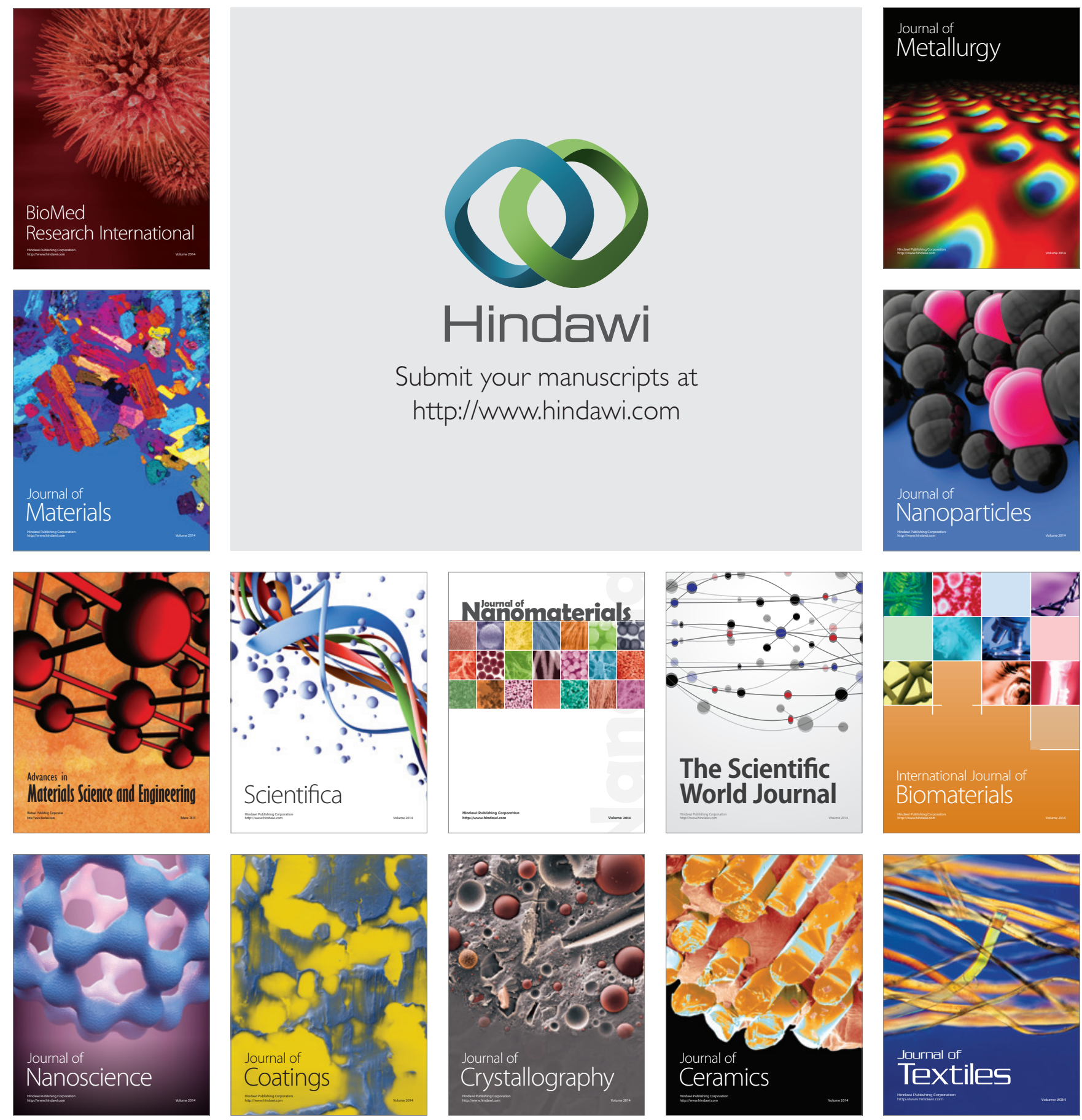